\title{
Inhibitory effects of Stichopus japonicus extract on melanogenesis of mouse cells via ERK phosphorylation
}

\author{
CHANG TAEK OH ${ }^{1 *}$, TAE-RIN KWON ${ }^{1 *}$, YU-JIN JANG $^{1,2}$, \\ KWANG HO YOO ${ }^{3}$, BEOM JOON KIM $^{1,2 *}$ and HEESU KIM ${ }^{3,4}$ \\ ${ }^{1}$ Department of Dermatology, Chung-Ang University College of Medicine; \\ ${ }^{2}$ Department of Medicine, Graduate School, Chung-Ang University, Seoul 156-756; \\ ${ }^{3}$ Department of Dermatology, College of Medicine, Catholic Kwandong University, International St. Mary's Hospital, \\ Incheon 22711; ${ }^{4}$ Department of Dermatology, Yonsei University College of Medicine, Seoul 03722, Republic of Korea
}

Received April 8, 2016; Accepted March 17, 2017

DOI: $10.3892 / \mathrm{mmr} .2017 .6686$

\begin{abstract}
Stichopus japonicus has been used as a folk medicine and as an ingredient in traditional food in East Asian countries. In recent years, the bioactive compounds found in $S$. japonicus have been reported to possess efficacy in wound healing and may be of potential use in the cosmeceutical, pharmaceutical and biomedical industries. Although the components and their functions require further investigation, S.japonicus extracts exhibit anti-inflammatory properties, and may be used for cancer prevention and treatment. Although several reports have examined different aspects of $S$. japonicus, the effects of $S$. japonicus extract on melanogenesis in the skin has not been reported to date. Therefore the present study aimed to investigate the effects of $S$. japonicus extract on melanogenesis. Treatment with a mixture of $S$. japonicus extracts (MSCE) reduced melanin synthesis and tyrosinase (TYR) activity in mouse melanocyte cells lines, B16F10 and Melan-A. In addition, MSCE treatment reduced the protein expression levels of TYR, tyrosinase-related protein-1 and tyrosinase-related protein-2. The reduced protein levels may be the result of decreased microphthalmia-associated transcription factor (MITF) expression, which is an important regulator of melanogenesis. The reduced expression level of MITF was associated with delayed phosphorylation of extracellular signal-regulated kinase (ERK) induced by MSCE treatment. A specific MEK inhibitor, PD98059, significantly blocked MSCE-mediated inhibition of melanin synthesis. In
\end{abstract}

Correspondence to: Professor Heesu Kim, Department of Dermatology, College of Medicine, Catholic Kwandong University, International St. Mary's Hospital, 404-834, Simgokro 100GIL $25 \mathrm{Seo}$, Incheon 22711, Republic of Korea

E-mail: yollikim@gmail.com

${ }^{*}$ Contributed equally

Key words: Stichopus japonicus, melanogenesis, mitogenactivated protein kinase 1, B16F10 cells, Melan-A cells conclusion, these results indicate that MSCE may be useful as a potential skin-whitening compound in the skin medical industry.

\section{Introduction}

Melanocytes are melanin-producing cells that are located in the basal layer of the skin epidermis. Melanogenesis is the physiological process that mediates photoprotection of the skin from ultraviolet (UV) radiation-induced injury by producing melanin in the skin and hair (1). However, overexposure to UV radiation may result in hyperpigmentation disorders, including melasma, freckles, lentigo, skin wrinkles and skin cancers, all of which are of medical and cosmetic interest (2-4).

Melanogenesis is regulated by melanogenic enzymes, including tyrosinase (TYR), tyrosinase-related protein 1 (TRP-1), and tyrosinase related protein 2 (TRP-2) (5). TYR is the predominant enzyme that catalyzes the rate-limiting phase of melanogenesis, and the inhibition of this enzyme is a typical method used to improve pigmentation (6). TYR is crucial for the regulation of melanin synthesis through the hydroxylation of tyrosine to 3,4-dihydroxyphenylalanine (DOPA) and the oxidation of DOPA to dopaquinone (7). Members of the tyrosinase (TYR) gene family (which includes TYR, TRP-1 and $T R P-2)$ that are responsible for melanin synthesis are transcriptionally regulated by microphthalmia-associated transcription factor (MITF), which regulates differentiation, proliferation and pigmentation of melanocytes. In addition, mutations in MITF can lead to the abnormal synthesis of melanin, which results in hyperpigmentation of the skin and hair (8). For this reason, compounds that inhibit the expression of MITF may be useful as depigmentation agents (9).

It is well known that various signaling pathways are involved in regulating melanogenesis. Phosphorylation of the mitogen-activated protein kinase (MAPK) signaling pathways lead to a reduction of melanin synthesis in mouse melanocytes. Several studies suggested that activation/phosphorylation of the extracellular signal-regulated kinase (ERK) and c-Jun N-terminal kinase (JNK) signaling pathways results in the inhibition of melanogenesis $(10,11)$. This effect has been attributed to the phosphorylation of MITF at serine 73, which 
results in MITF ubiquitination and degradation (12). Thus, the activation of ERK and JNK signaling inhibits melanogenesis by reducing MITF expression and TYR activity.

A number of melanin synthesis inhibitors, including kojic acid and arbutin, have been the focus of previous studies and are currently being used as functional bio-cutaneous compounds for whitening of pigmentary disorders such as melasma, dark circles and post-inflammatory hyperpigmentation (13). Compounds with possible applications in the cosmetic industry have been the target of ongoing research and attention. However, the use of melanogenesis inhibitors is heavily regulated owing to their clinical side effects, and only a few of them (such as arbutin and kojic acid) are currently used commercially (14). Therefore, it would be beneficial to identify additional effective compounds that may be able to modulate melanogenesis for use in the medical and cosmetic industry.

The Korean red sea cucumber Stichopus japonicus is a marine echinoderm of the Holothuroidea family; it is consumed in East Asian countries, such as China, Indonesia, Korea and Japan. For centuries, it has been used as a remedy for inflammation and to treat illnesses or infections from viral and bacterial pathogens (15). S. japonicus has also been used as an effective remedy for treating various internal and external wounds, including athlete's foot. Several studies have demonstrated that $S$. japonicus extracts exhibit a number of biological properties, such as antioxidant, antifungal, and immunomodulatory activity (16-18). These extracts have been revealed to contain lectins, peptides, minerals, vitamins and various other molecules, including chondroitin sulfates, glycosaminoglycan and polysaccharides. These compounds may be responsible for the powerful wound-repairing abilities, and anti-inflammatory, antifungal, and antiviral properties (19). Based on these data, S. japonicus extracts may be an excellent candidate for inclusion in cosmeceutical and skin medical compounds for treating skin pigmentation disorders including melasma and post-inflammatory hyperpigmentation (PIH).

However, depigmentation via inhibition of TYR and melanin synthesis, and the associated molecular mechanism of the mixture of $S$. japonicus extracts (MSCE), have not been examined as a candidate for a skin topical agent to improve hyperpigmentation. To investigate the mechanistic action of MSCE in melanin synthesis, the effects of MSCE on melanin synthesis and TYR expression was investigated. The results suggested that MSCE treatment inhibited melanogenesis through the ERK signaling pathway in mouse melanocyte cells.

\section{Materials and methods}

Sample preparation and extraction procedure. Over 1,000 live specimens of $S$. japonicus (average body weight $180 \mathrm{~g}$ ) were obtained from fishermen on the Korean islands of Sochong and Daechong. Fresh $S$. japonicus specimens were divided into the visceral organs and the body, and were rinsed with clean water. Subsequently, the body was dried using traditional salting and drying procedures, which involved drying for 1 day in the sea breeze, and the visceral organs were frozen at -80 to- $90^{\circ} \mathrm{C}$. The dried $S$. japonicus bodies, which were prepared at Catholic Kwandong University, International St. Mary's
Hospital (Incheon, Korea), were ground using a blender and $150 \mathrm{~g}$ of the resulting powder was dissolved in 2 liters $65 \%$ ethanol for $2 \mathrm{~h}$ at room temperature with continual mixing. The solution was filtered through cotton and underwent reflux extraction using a COSMOS-660 Universal Vacuum Extractor (Kyungseo Machine Co., Incheon, Korea). The extract was concentrated using an EYELA N-12 Vacuum Evaporator connected to an EYELA CA-1112 Low-Temperature Water Circulator (Tokyo Rikakikai Co., Ltd., Tokyo, Japan) prior to freeze-drying with a TFD-100 Freeze Dryer (ilShinBioBase Co., Ltd., Gyeonggi-do, Korea) and powdered.

Materials. Mushroom TYR, $\alpha$-melanocyte stimulating hormone ( $\alpha$-MSH), L-DOPA, phorbol 12-myristate 13-acetate (PMA), phenylthiourea (PTU), kojic acid and the MAPK/ERK kinase inhibitor PD98059 were purchased from Sigma-Aldrich (Merck KGaA, Darmstadt, Germany). The Cell Counting Kit-8 (CCK-8) was purchased from Dojindo Molecular Technologies, Inc. (Kumamoto, Japan). Dulbecco's modified Eagle's medium (DMEM), phosphate-buffered saline (PBS), Roswell Park Memorial Institute (RPMI)-1640 medium, and penicillin/streptomycin $(\mathrm{P} / \mathrm{S})$ were purchased from WelGene, Inc. (Daegu, Korea). Fetal bovine serum (FBS) was purchased from Gibco (Thermo Fisher Scientific, Inc., Waltham, MA, USA). Primary antibodies against ERK (\#9102), phosphorylated (p)-ERK (\#9101), JNK (\#9252), p-JNK (\#9251), p38 (\#9212) and p-p38 MAPK (\#9211) were purchased from Cell Signaling Technology, Inc. (Danvers, MA, USA). Antibodies against TYR (SC-7833), TRP-1 (SC-10443), TRP-2 (SC-74439) and $\beta$-actin (SC-47778) were purchased from Santa Cruz Biotechnology, Inc. (Dallas, TX, USA). The antibody against MITF (MA5-14146) was purchased from NeoMarkers, Inc. (Portsmouth, NH, USA). Secondary antibodies specific for horseradish peroxidase (HRP) goat anti-rabbit immunoglobulin (Ig)G (cat. no. PI-1000), HRP horse anti-mouse IgG (cat. no. PI-2000), and HRP horse anti-goat IgG (cat. no. PI-9500) were purchased from Vector Laboratories, Inc. (Burlingame, CA, USA).

Cell culture. B16F10 mouse melanoma cells were purchased from the American Type Culture Collection (Manassas, VA, USA). Cells were cultured in DMEM containing 10\% FBS and $1 \% \mathrm{P} / \mathrm{S}$ in $5 \% \mathrm{CO}_{2}$ at $37^{\circ} \mathrm{C}$. Melan-A immortalized mouse melanocytes were obtained from Professor Dorothy C. Bennett (St. George's, University of London, London, UK) (20) and were cultured in RPMI-1640 medium containing 10\% FBS, $1 \% \mathrm{P} / \mathrm{S}$, and $200 \mathrm{nM}$ PMA in $10 \% \mathrm{CO}_{2}$ at $37^{\circ} \mathrm{C}$.

Cell viability assay. The mouse cells were seeded $\left(1 \times 10^{4}\right.$ cells/well) in 96-well plates and incubated at $37^{\circ} \mathrm{C}$. Following a $24 \mathrm{~h}$ incubation, the cells were washed with DPBS (WelGene, Inc., Daegu, Korea) and replaced with DMEM (B16F10 cells) or RPMI1640 (Melan-A cells), containing MSCE diluted to various concentrations at 0-100\%. Following $24 \mathrm{~h}$ at $37^{\circ} \mathrm{C}$, the cells were washed with DPBS, and DMEM (B16F10 cells) or RPMI1640 (Melan-A cells) containing $10 \%$ CCK-8 solution was added. The cells were then incubated at room temperature and the absorbance was measured at $450 \mathrm{~nm}$ using a microplate reader (SpectraMax 340; Molecular Devices, LLC, Sunnyvale, CA, USA). 
Mushroom TYR activity assay. A mushroom TYR activity assay was assessed by measuring DOPA oxidase activity using a slightly modified experimental protocol from a previous study (21). Briefly, $95 \mu \mathrm{l}$ of $0.1 \mathrm{M}$ sodium phosphate buffer ( $\mathrm{pH}$ 6.5) containing the indicated concentrations of kojic acid $(50 \mu \mathrm{M})$ and MSCE, and $20 \mu \mathrm{l}$ of mushroom TYR $(1,000 \mathrm{U} / \mathrm{ml})$ were combined in each well, and $20 \mu \mathrm{l}$ of $5 \mathrm{mM}$ L-DOPA was then added. The absorbance was measured at $475 \mathrm{~nm}$ every $10 \mathrm{~min}$ for $1 \mathrm{~h}$ using a microplate reader (SpectraMax 340; Molecular Devices, LLC, Sunnyvale, CA, USA).

Melanin assay. B16F10 cells characteristically release melanin, which was measured with slight modifications to a previously described protocol (22). Briefly, cells were seeded $\left(1 \times 10^{5}\right.$ cells/well) in 6 -well plates. Following $24 \mathrm{~h}$ at $37^{\circ} \mathrm{C}$, the plates were washed with DPBS and the media was replaced with phenol red-free DMEM containing $\alpha$-MSH $(1 \mu \mathrm{M})$, or PD98059 $(10 \mu \mathrm{M})$ for $30 \mathrm{~min}$ at $37^{\circ} \mathrm{C}$ followed by $\alpha$-MSH and MSCE treatment as aforementioned. The cells were subsequently incubated for 3 days. Absorbance was measured in the cell culture supernatant at $405 \mathrm{~nm}$ using a microplate reader (SpectraMax 340; Molecular Devices, LLC, Sunnyvale, CA, USA). PTU is an inhibitor of melanin synthesis, and this compound was employed as a positive control; BF16F10 cells were treated with PTU $(50 \mu \mathrm{M})$ for 3 days at $37^{\circ} \mathrm{C}$ in place of MSCE.

Melan-A cells were seeded $\left(5 \times 10^{5}\right.$ cells/well) in 24 -well plates. Following $24 \mathrm{~h}$ incubation, the plates were washed with DPBS, and then RPMI1640 containing the indicated concentrations of MSCE or PD98059 (pretreated for $30 \mathrm{~min}$ at $37^{\circ} \mathrm{C}$, prior to MSCE treatment) was added. The plates were subsequently incubated at $37^{\circ} \mathrm{C}$ for 3 days. Cells were lysed in $110 \mu \mathrm{l}$ of $1 \mathrm{~N} \mathrm{NaOH}$ at $55^{\circ} \mathrm{C}$ for $15 \mathrm{~min}$, and the absorbance was measured at $470 \mathrm{~nm}$ using a microplate reader (SpectraMax 340; Molecular Devices, LLC, Sunnyvale, CA, USA). All results were normalized to the total protein concentration of the cell pellet using a Bicinchoninic Acid Assay kit (Pierce; Thermo Fisher Scientific, Inc.).

Western blot analysis. Cells were cultured in a $100 \mathrm{~mm}$ dish and treated as aforementioned for 0-720 min. Cells were lysed using a cell lysis buffer [62.5 mM Tris- $\mathrm{HCl}$ (pH 6.8), $2 \%$ SDS, $5 \%$ $\beta$-mercaptoethanol, $2 \mathrm{mM}$ phenylmethylsulfonyl fluoride, Complete Protease Inhibitors (Roche Diagnostics $\mathrm{GmbH}$, Mannheim, Germany), $1 \mathrm{mM} \mathrm{Na} \mathrm{VO}_{4}, 50 \mathrm{mM} \mathrm{NaF}$ and $10 \mathrm{mM}$ EDTA] and centrifuged at $13,000 \mathrm{x} \mathrm{g}$ for $20 \mathrm{~min}$ at $4^{\circ} \mathrm{C}$. Equal amounts $(20 \mu \mathrm{g})$ of protein were separated by SDS-PAGE (8-12\%) and transferred onto a polyvinylidene fluoride membrane (EMD Millipore, Billerica, MA, USA). Following blocking with 5\% skim milk for $2 \mathrm{~h}$ at room temperature, the membranes were probed with primary antibodies $(1: 1,000)$ for $24 \mathrm{~h}$ at $4^{\circ} \mathrm{C}$ and then incubated with horseradish peroxidase-conjugated secondary antibodies $(1: 1,000)$ for $3 \mathrm{~h}$ at room temperature. Following washing in TBS$0.1 \%$ Tween-20 (TBS-T) buffer, hybridized antibodies were detected using an Enhanced Chemiluminescence detection kit (Amersham; GE Healthcare Life Sciences, Chalfont, UK) and images were captured using an LAS-1000 Lumino-image Analyzer (Fujifilm, Tokyo, Japan).
Immunocytochemistry. Cells were seeded ( $1 \times 10^{5}$ cells/well) onto glass coverslips pre-coated with poly-L-lysine (Sigma-Aldrich; Merck KGaA) and incubated for $24 \mathrm{~h}$ at $4^{\circ} \mathrm{C}$. Slides were fixed with $4 \%$ paraformaldehyde for $15 \mathrm{~min}$ at room temperature. Following washing with PBS, cells were treated with $0.01 \%$ Triton X-100 (Sigma-Aldrich; Merck $\mathrm{KGaA}$ ) and then blocked with $2 \%$ bovine serum albumin (Merck KGaA) in TBS-T for $1 \mathrm{~h}$ at room temperature. The cells were incubated with an anti-TYR antibody $(1: 100)$ at $4^{\circ} \mathrm{C}$ overnight. Following washing with TBS-T $+0.1 \%$ Tween-20 buffer, cells were incubated for $3 \mathrm{~h}$ at $4^{\circ} \mathrm{C}$ with goat anti-rabbit IgG-fluorescein isothiocyanate secondary antibodies $(1: 1,000$; SC-2012, Santa Cruz Biotechnology, Inc.) and mounted with fluorescent mounting medium containing DAPI(Golden Bridge International, Inc., Bothell, WA, USA). Cell morphology was observed using a FluoView FV10i fluorescence microscope and a DP70 Digital Microscope Camera (both from Olympus Corporation, Tokyo, Japan). Images were processed using the proprietary DP70 Controller software (Ver.03.03; Olympus Corporation).

Statistical analysis. Statistical analyses were performed using SPSS 18.0 (SPSS, Inc., Chicago, IL, USA). There were three replicates for each of the data measurements. Results are presented as the mean \pm standard deviation. Data were analyzed by one-way analysis of variance, followed by Tukey's multiple comparison tests. $\mathrm{P}<0.05$ was considered to indicate a statistically significant difference.

\section{Results}

Effects of MSCE on mouse melanocyte cell viability. First, a CCK-8 assay was performed to evaluate the cytotoxicity of MSCE on B16F10 mouse melanoma cells and Melan-A normal mouse melanocytes. Cells were treated with MSCE at a concentration range of $0-100 \%$. As demonstrated in Fig. 1, there was no detectable cytotoxicity at concentrations ranging from 0.78-6.25\%. Therefore, the MSCE concentration range of $1.25-5 \%$ was used for the subsequent experiments.

Effects of MSCE on melanogenesis in B16F10 and Melan-A cells. The effects of MSCE treatment on melanogenesis in mouse cells were examined. To determine whether MSCE inhibits melanin production in mouse cells, melanin content was measured following 3 days treatment with MSCE at concentrations of $1.25-5 \%$. In cells treated with $\alpha-\mathrm{MSH}$, melanin levels in the media increased compared with untreated cells, due to an induction of melanogenesis (Fig. 2A). However, cells co-treated MSCE exhibited a dose-dependent reduction in melanin pigmentation in B16F10 and Melan-A cells, compared with cells treated with $\alpha-\mathrm{MSH}$ alone (Fig. 2A). PTU, a well-known TYR inhibitor (4), was used as a positive control; PTU treatment inhibited melanin pigmentation compared with cells treated with $\alpha$-MSH alone (Fig. 2A).

Melanogenesis is regulated by an enzymatic cascade that is under the control of TYR (23). For this reason, various compounds that have been developed for skin-whitening purposes directly inhibit TYR (24). The effect of MSCE on TYR activity was examined using a mushroom TYR activity assay. Treatment with $50 \mu \mathrm{M}$ kojic acid, a direct inhibitor of 

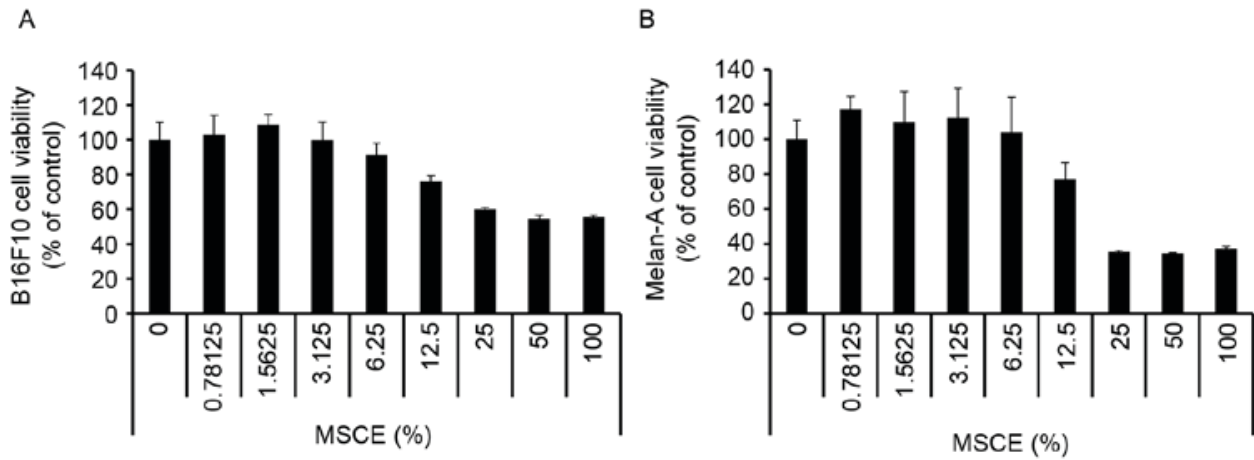

Figure 1. Effects of MSCE treatment on mouse melanocyte cell viability. (A) B16F10 mouse melanoma cells and (B) Melan-A mouse melanocytes were treated with MSCE at concentrations ranging from 0-100\% for $24 \mathrm{~h}$ in serum-free media. Cell viability was then measured by Cell Counting Kit-8 assay. Each experiment was conducted in triplicate and the data are presented as the mean \pm standard deviation. MSCE, mixed Stichopus japonicus extract.
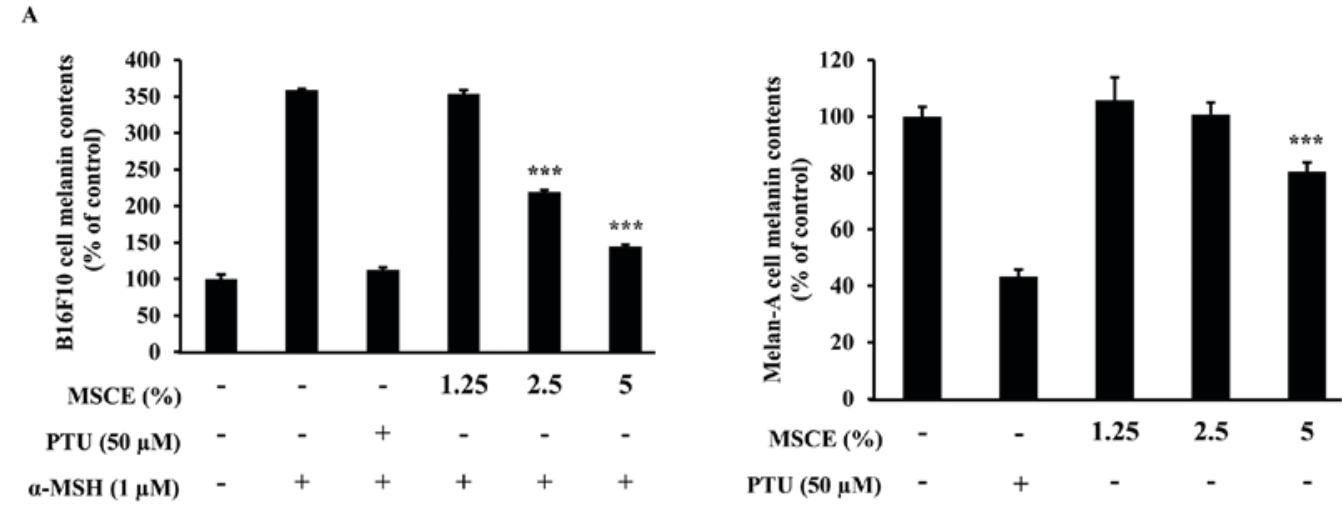

B

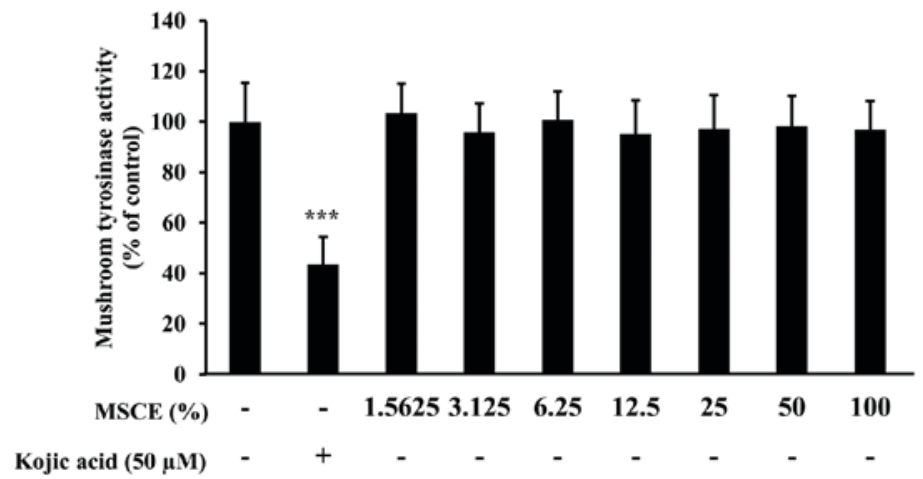

Figure 2. Effects of MSCE treatment on melanogenesis in mouse melanocyte cells. (A) B16F10 mouse melanoma and melan-A mouse melanocyte cells were incubated with MSCE (1.25-5\%) or PTU $(50 \mu \mathrm{M})$ for 3 days, and melanin content was measured. B16F10 cells were additionally induced towards melanogenesis with $1 \mu \mathrm{M} \alpha$-MSH. (B) Mushroom TYR activity was measured in the presence of MSCE (0-100\%). Kojic acid, a known inhibitor of TYR activity, was used as a positive control. Each experiment was conducted in triplicate and the data are presented as the mean \pm standard deviation. ${ }^{* * *} \mathrm{P}<0.001 \mathrm{vs}$. $\alpha$-MSH-treated B16F10 cells or untreated Melan-A cells. $\alpha$-MSH, $\alpha$-melanocyte stimulating hormone; MSCE, mixed Stichopus japonicus extract; PTU, phenylthiourea; TYR, tyrosinase.

TYR that was used in this assay as a positive control, resulted in significant inhibition of TYR activity (Fig. 2B). However, treatment with various concentrations of MSCE did not inhibit mushroom TYR activity (Fig. 2B). These results demonstrated that the inhibitory effect of MSCE on melanogenesis was not mediated by the direct inhibition of TYR.

Effects of MSCE on melanogenesis signaling pathways in B16F10 and Melan-A cells. To investigate whether the inhibitory effect of MSCE is related to the melanogenesis signaling pathways, mouse cells were treated with $\alpha-\mathrm{MSH}$ and/or MSCE, and the protein expression levels of TYR, TRP-1, TRP-2 and MITF was examined by western blotting. As demonstrated in Fig. 3A, the protein expression levels of TYR, TRP-1 and TRP-2 were reduced following co-treatment with MSCE for 24,48 or $72 \mathrm{~h}$, compared with the $\alpha$-MSH-treated group in B16F10 cells. In addition, MITF protein expression levels were reduced in a time-dependent manner compared with the $\alpha$-MSH-treated group in B16F10 cells (Fig. 3A). These results indicated that MSCE treatment inhibited melanin production 
A

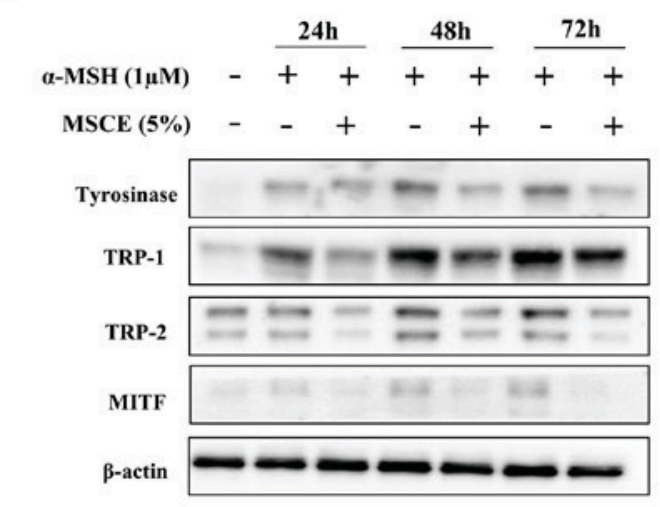

C

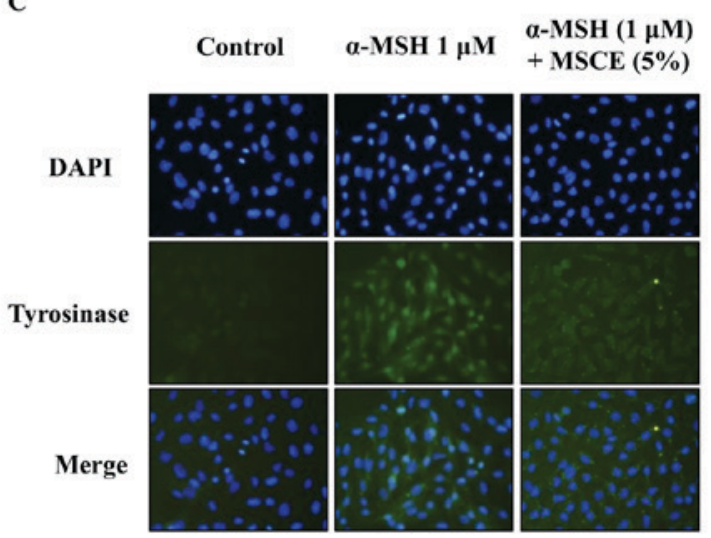

B

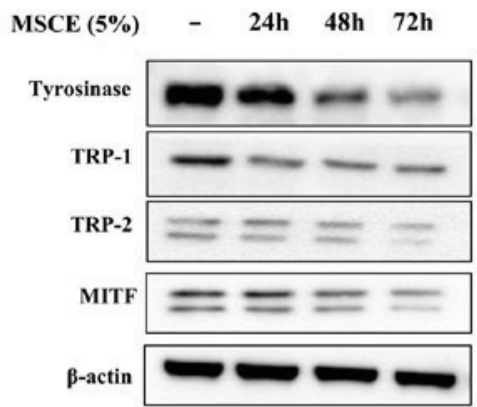

D

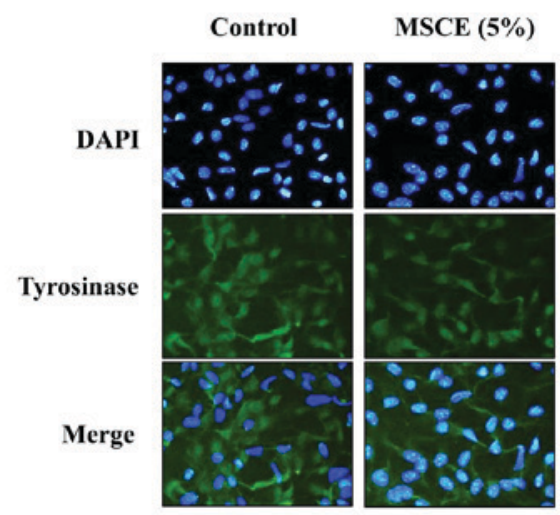

Figure 3. Effects of MSCE on melanogenesis-related proteins. B16F10 mouse melanoma cells (left panels) and Melan-A mouse normal melanocytes (right panels) were treated with MSCE (5\%) for 24, 48 or $72 \mathrm{~h}$. B16F10 cells were additionally induced towards melanogenesis with $1 \mu \mathrm{M} \alpha$-MSH. (A) Cell lysates were analyzed by western blotting for TYR, TRP-1, TRP-2 and MITF protein expression. $\beta$-actin was used as loading control. (B) Expression of TYR was also examined by immunocytochemical staining. Representative images of (C) B16F10 cells and (D) Melan-A melanocytes from experiments performed in triplicate depict TYR expression (green), nuclear DAPI staining (blue) and merged signals (magnification, x200). $\alpha$-MSH, $\alpha$-melanocyte stimulating hormone; MITF, microphthalmia-associated transcription factor; MSCE, mixed Stichopus japonicus extract; TRP, tyrosinase related protein; TYR, tyrosinase.

through the reduction of MITF and TYR expression levels. The inhibitory effect of MSCE on TYR expression in the mouse cell lines was confirmed by immunocytochemistry. The results indicated that TYR levels were reduced following MSCE treatment for $72 \mathrm{~h}$, compared with the $\alpha$-MSH-treated group in B16F10 cells and with the untreated group in Melan-A cells (Fig. 3B).

Effects of MSCE on signal transduction pathways in mouse cells. In an attempt to identify the underlying mechanisms involved in the MSCE-mediated cell depigmentation, the effects of MSCE treatment (5\%) on the activation of ERK, JNK and p38 MAPKs were examined by western blot analysis. In B16F10 cells treated with MSCE for up to $720 \mathrm{~min}$, ERK and JNK phosphorylation was greatly increased at the 30 and 60 min time points, respectively (Fig. 4A). By contrast, p38 phosphorylation increased in expression slightly (Fig. 4A). In Melan-A cells, ERK phosphorylation was notably increased at 10 min following MSCE treatment, whereas p38 phosphorylation was slightly increased at $120 \mathrm{~min}$. However, no change to JNK levels (Fig. 4B). To determine the possible involvement of p38 and JNK signaling on the MSCE-mediated inhibitory effect, cells were treated with the JNK and p38 inhibitors
(SP600125 and SB203580, respectively) and melanin production was analyzed following MSCE treatment. The results revealed no effect of the inhibitors on melanin production following MSCE treatment (data not shown).

Effects of MSCE on melanogenesis and ERK phosphorylation. The phosphorylation of ERK has been reported to inhibit TYR expression, which subsequently reduces cellular melanin production (25). Therefore, the present study examined whether the MSCE-induced activation of the ERK signaling pathway may be important for the MSCE-mediated inhibition of melanin production in B16F10 and Melan-A cells. For this purpose, mouse melanocyte cells were treated with MSCE for $30 \mathrm{~min}$ in the presence or absence of PD98059, a MEK inhibitor. As demonstrated in Fig. 5A, the melanin contents of B16F10 cells co-treated with $\alpha$-MSH and PD98059 were higher compared with cells treated with $\alpha$-MSH alone. Melan-A cells treated with PD98059 did not demonstrate any significant difference in melanin levels (data not shown). This synergistic effect of $\alpha$-MSH and PD98059 on melanin content was reduced by MSCE treatment (Fig. 5A). The effects of PD98059 treatment on MSCE-induced ERK phosphorylation was examined by western blotting, which 
A

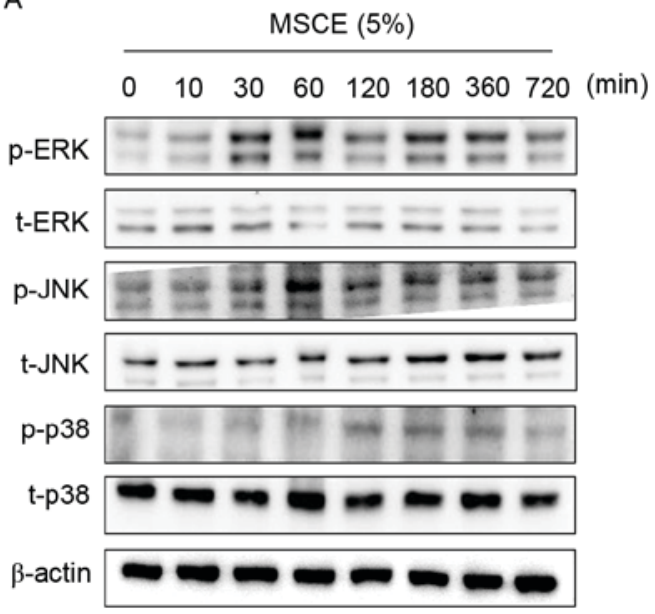

B

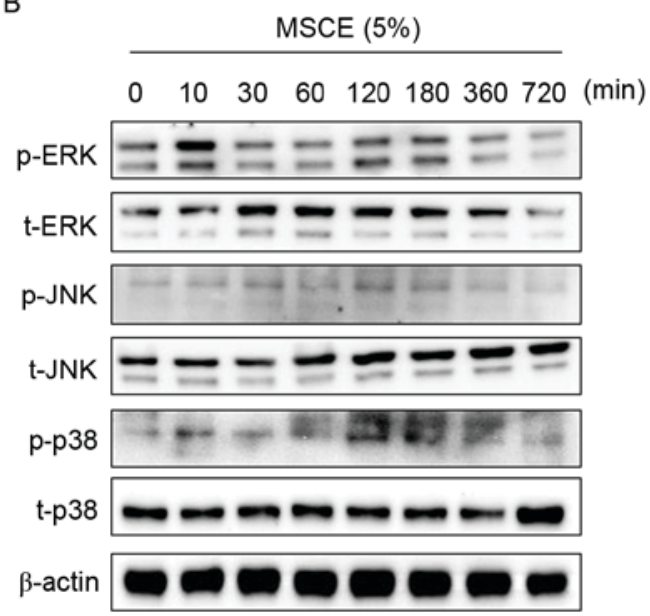

Figure 4. Effects of MSCE on signal transduction pathways in mouse melanocyte cells. Following $24 \mathrm{~h}$ of serum starvation, (A) B16F10 mouse melanoma cells and (B) Melan-A mouse normal melanocytes were treated with 5\% MSCE for the indicated time periods. Cell lysates were harvested for western blot analysis using primary antibodies against p-ERK, t-ERK, p-JNK, t-JNK, p-p38 and t-p38; $\beta$-actin was used as the loading control. The results are representative of triplicate experiments. $\alpha$-MSH, $\alpha$-melanocyte stimulating hormone; ERK, extracellular signal-regulated kinase; JNK, c-Jun N-terminal kinase; MSCE, mixed Stichopus japonicus extract; $\mathrm{p}$, phosphorylated; $\mathrm{p} 38$, mitogen-activated protein kinase 14 ; $\mathrm{t}$, total.
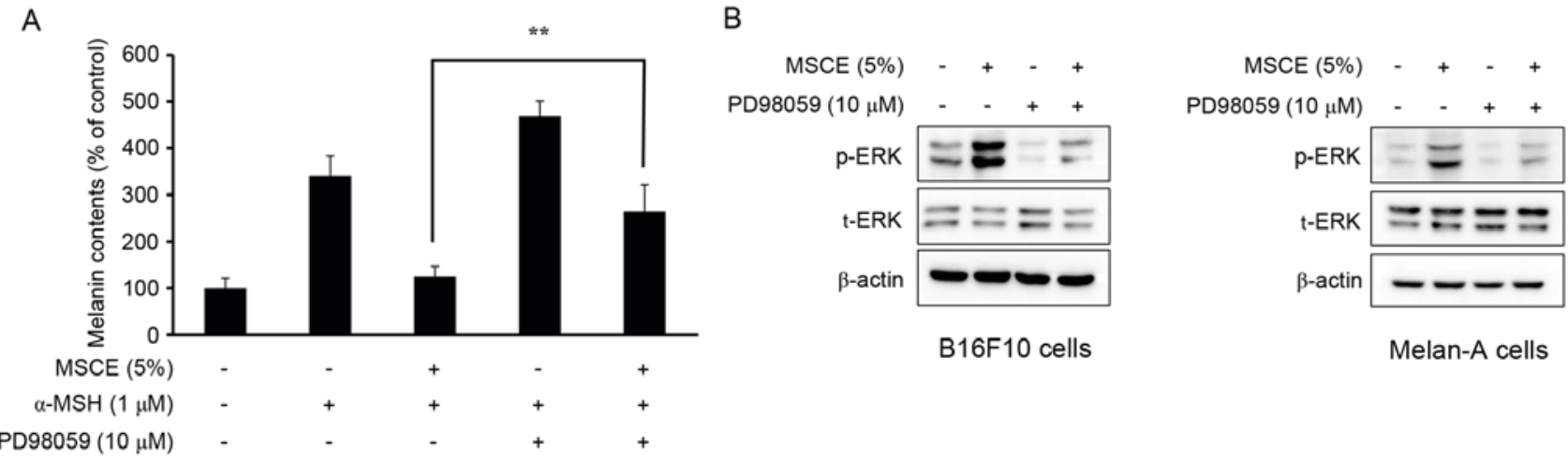

Figure 5. Effects of MSCE on ERK phosphorylation and melanogenesis. (A) B16F10 mouse melanoma cells were treated with 5\% MSCE in the presence or absence of PD98059 $(10 \mu \mathrm{M})$ after addition of $\alpha$-MSH $(1 \mu \mathrm{M})$ for 3 days, and melanin content was measured. Results are presented as the mean \pm standard deviation and each experiment was performed in triplicate. ${ }^{* *} \mathrm{P}<0.01$. (B) B16F10 cells and Melan-A mouse normal melanocytes were treated with MSCE (5\%) for $30 \mathrm{~min}$ in the presence or absence of PD98059 $(10 \mu \mathrm{M})$. Cell lysates were analyzed by western blot for p-ERK and t-ERK. $\beta$-actin was used as the loading control. The results are representative of triplicate experiments. $\alpha$-MSH, $\alpha$-melanocyte stimulating hormone; ERK, extracellular signal-regulated kinase; MSCE, mixed Stichopus japonicus extract; p, phosphorylated; PD98059, MEK inhibitor; t, total.

revealed a reduction of ERK phosphorylation levels in the MSCE-treated mouse cells following PD98059 addition (Fig. 5B). These results indicated that ERK phosphorylation may be involved in the MSCE-mediated reduction of melanogenesis.

\section{Discussion}

Pigmentary disorders, including melasma, lentigo, post-inflammatory hyperpigmentation and dark circles, are associated with abnormal regulations of melanin synthesis or melanosome transfer (26). These disorders irregularly darken the skin of patients, and may cause discomfort or nervousness in everyday life. Several depigmentation compounds have been created and have both medical and clinical applications in skin. However, many of these compounds produce toxic and unwanted side effects owing to the inclusion of potentially harmful ingredients, such as mercury and high levels of hydroquinone (27). For this reason, research has been focused on the development of safe and effective alternative whitening compounds. Various natural products originating from marine organisms and plants have been investigated for effects on pigmentation, including TYR, TRP-1 and TRP-2 expression, and are used as traditional whitening compounds (28). The present study demonstrated the inhibitory effects of MSCE on melanin synthesis in B16F10 and Melan-A cells and its underlying mechanism.

Yoon et al (29) previously reported an effect of sea cucumber on the expression of several melanogenic proteins, including TYR, TRP-1, TRP-2 and MITF. Furthering this research, the present study investigated the mechanism of MSCE as a novel skin-whitening compound. The results further established that MSCE functions via the inhibition of TYR, TRP-1, TRP-2 and MITF protein expression. The effects of MSCE were dose-dependent and did not produce any critical cytotoxicity at doses between 1.25 and $5 \%$ that were used for the melanogenesis assays. TYR has been reported to 
catalyze the rate-limiting phase of melanin synthesis, and it is the first target of PTU, a compound with important depigmentation effects that was recently employed for medical and cosmetic purposes (30). MSCE treatment did not significantly alter TYR activity when tested on mushroom TYR; however, MSCE treatment did significantly decrease melanin synthesis in B16F10 and Melan-A cells in a dose-dependent manner. These results suggested that MSCE did not directly inhibit TYR activity, indicating that the effects may be a result of indirect mechanisms. Thus, the MSCE-induced depigmentation may be the result of inhibitory effects on the signaling pathways controlling TYR expression.

To understand the results of the present study, the mechanisms involved in MSCE-mediated melanogenesis inhibition were investigated. Western blot analysis of melanogenesis-related proteins was performed, and the results suggested that MSCE treatment reduced the protein expression levels of TYR, TRP-1, TRP-2 and MITF in a time-dependent manner. In addition, MSCE treatment reduced the melanin content compared with untreated cells. The effects of MSCE on TYR expression, which regulates melanin synthesis in B16F10 and Melan-A cells, were confirmed using immunocytochemical analysis. The results indicated that the TYR protein expression levels in B16F10 cells and Melan-A cells were reduced following treatment with MSCE for $72 \mathrm{~h}$ compared with untreated cells.

The MAPKs, including ERK, JNK and p38 MAPK, are important in regulating melanogenesis (31). To explain the mechanisms underlying the depigmentation effects of MSCE treatment, changes to the activation/phosphorylation of ERK, JNK and p38 MAPK were examined in a time-course MSCE treatment experiment using western blot analysis for 0-720 min. The results demonstrated that the phosphorylation of ERK was significantly increased and phosphorylation of JNK was slightly increased following MSCE treatment compared with untreated cells; however, p38 MAPK phosphorylation remained unchanged. These findings suggested that MSCE-induced depigmentation in B16F10 and Melan-A cells may occur through MAPK-regulated signaling pathways. To identify which MAPKs may be mediating MSCE-induced melanogenesis inhibition, B16F10 and Melan-A cells were analyzed in the presence of SB203580, a p38 inhibitor, and PD98059, a specific MEK inhibitor. Although SB203580 had no effect in melanin production (data not shown), PD98059 markedly increased MSCE-suppressed melanin contents in B16F10 cells. In addition, the PD98059-induced suppression of p-ERK expression was reversed by MSCE treatment in $\alpha$-MSH-treated B16F10 and Melan-A cells. Notably, it has previously been reported that ERK is an important regulator of melanogenesis, as ERK phosphorylation induces MITF activation and its following degradation, and thus, inhibits melanin synthesis. In addition, the phosphorylation of ERK inhibits melanin synthesis through the suppression of TYR expression (32). The effects of MSCE on melanogenesis and phosphorylation of ERK demonstrated in the present study are in accordance with the known role of the ERK signaling pathway in melanin synthesis.

In conclusion, the present study demonstrated that MSCE treatment led to the phosphorylation of ERK and JNK, which suppressed the degradation of MITF, TYR, TRP-1, and TRP-2 in B16F10 and Melan-A cells, subsequently reducing melanin synthesis. Therefore, the results suggested that MSCE may be a useful depigmenting compound that may be used in the treatment of unwanted pigmentation conditions and may be a novel future therapeutic in the medical and cosmetic industries.

\section{Acknowledgements}

The present study was supported by research funding from Catholic Kwandong University International, St. Mary's Hospital (grant no. CKURF-201406830001).

\section{References}

1. D'Mello SA, Finlay GJ, Baguley BC and Askarian-Amiri ME: Signaling pathways in melanogenesis. Int J Mol Sci 17: pii: E1144, 2016.

2. Ichihashi M and Ando $\mathrm{H}$ : The maximal cumulative solar UVB dose allowed to maintain healthy and young skin and prevent premature photoaging. Exp Dermatol 23 (Suppl 1): S43-S46, 2014.

3. Sklar LR, Almutawa F, Lim HW and Hamzavi I: Effects of ultraviolet radiation, visible light, and infrared radiation on erythema and pigmentation: A review. Photochem Photobiol Sci 12: 54-64, 2013.

4. Gange RW: Comparison of pigment responses in human skin to UVB and UVA radiation. Prog Clin Biol Res 256: 475-485, 1988

5. Huang HC, Hsieh WY, Niu YL and Chang TM: Inhibitory effects of adlay extract on melanin production and cellular oxygen stress in B16F10 melanoma cells. Int J Mol Sci 15: 16665-16679, 2014.

6. Ma H, Xu J, DaSilva NA, Wang L, Wei Z, Guo L, Johnson SL, $\mathrm{Lu} \mathrm{W}, \mathrm{Xu} \mathrm{J}, \mathrm{Gu} \mathrm{Q}$ and Seeram NP: Cosmetic applications of glucitol-core containing gallotannins from a proprietary phenolic-enriched red maple (Acer rubrum) leaves extract: Inhibition of melanogenesis via down-regulation of tyrosinase and melanogenic gene expression in B16F10 melanoma cells. Arch Dermatol Res 309: 265-274, 2017.

7. Camacho-Hübner A and Beermann F: Cellular and molecular features of mammalian pigmentation-tyrosinase and TRP. Pathol Biol (Paris) 48: 577-583, 2000 (In French).

8. Ishii N, Ryu M and Suzuki YA: Lactoferrin inhibits melanogenesis by down-regulating MITF in melanoma cells and normal melanocytes. Biochem Cell Biol 95: 119-125, 2017.

9. Tagashira H, Miyamoto A, Kitamura S, Tsubata M, Yamaguchi K, Takagaki K and Imokawa G: UVB stimulates the expression of endothelin $\mathrm{B}$ receptor in human melanocytes via a sequential activation of the p38/MSK1/CREB/MITF pathway which can be interrupted by a french maritime pine bark extract through a direct inactivation of MSK1. PLoS One 10: e0128678, 2015.

10. Han JS, Sung JH and Lee SK: Antimelanogenesis activity of hydrolyzed ginseng extract (GINST) via Inhibition of JNK Mitogen-activated protein kinase in B16F10 Cells. J Food Sci 81: H2085-H2092, 2016.

11. Huang HC, Wei CM, Siao JH, Tsai TC, Ko WP, Chang KJ, Hii CH and Chang TM: Supercritical fluid extract of spent coffee grounds attenuates melanogenesis through downregulation of the PKA, PI3K/Akt, and MAPK signaling pathways. Evid Based Complement Alternat Med 2016: 5860296, 2016.

12. Fu YT, Lee CW, Ko HH and Yen FL: Extracts of Artocarpus communis decrease $\alpha$-melanocyte stimulating hormone-induced melanogenesis through activation of ERK and JNK signaling pathways. ScientificWorldJournal 2014: 724314, 2014.

13. Hseu YC, Cheng KC, Lin YC, Chen CY, Chou HY, Ma DL, Leung CH, Wen ZH and Wang HM: Synergistic effects of linderanolide B Combined with Arbutin, PTU or kojic acid on tyrosinase inhibition. Curr Pharm Biotechnol 16: 1120-1126, 2015.

14. Desmedt B, Courselle P, De Beer JO, Rogiers V, Grosber M, Deconinck E and De Paepe K: Overview of skin whitening agents with an insight into the illegal cosmetic market in Europe. J Eur Acad Dermatol Venereol 30: 943-950, 2016.

15. Wang X, Wang L, Che J, Li Z, Zhang J, Li X, Hu W and $\mathrm{Xu}$ Y: Improving the quality of Laminaria japonica-based diet for Apostichopus japonicus through degradation of its algin content with Bacillus amyloliquefaciens WB1. Appl Microbiol Biotechnol 99: 5843-5853, 2015. 
16. Liu X, Sun Z, Zhang M, Meng X, Xia X, Yuan W, Xue F and Liu C: Antioxidant and antihyperlipidemic activities of polysaccharides from sea cucumber Apostichopus japonicus. Carbohydr Polym 90: 1664-1670, 2012.

17. Gao F, Li F, Tan J, Yan J and Sun H: Bacterial community composition in the gut content and ambient sediment of sea cucumber Apostichopus japonicus revealed by $16 \mathrm{~S}$ rRNA gene pyrosequencing. PLoS One 9: e100092, 2014.

18. Himaya SW, Ryu B, Qian ZJ and Kim SK: Sea cucumber, Stichopus japonicus ethyl acetate fraction modulates the lipopolysaccharide induced iNOS and COX-2 via MAPK signaling pathway in murine macrophages. Environ Toxicol Pharmacol 30: 68-75, 2010.

19. Zohdi RM,Zakaria ZA, Yusof N, Mustapha NM and Abdullah MN: Sea cucumber (Stichopus hermanii) based hydrogel to treat burn wounds in rats. J Biomed Mater Res B Appl Biomater 98: 30-37, 2011.

20. Bennett DC, Cooper PJ and Hart IR: A line of non-tumorigenic mouse melanocytes, syngeneic with the B16 melanoma and requiring a tumour promoter for growth. Int J Cancer 39: 414-418, 1987.

21. Shin HJ, Oh CT, Kwon TR, Beak HS, Joo YH, Kim JH, Lee CS, Lee JH, Kim BJ, Shin SS and Park ES: A novel adamantyl benzylbenzamide derivative, AP736, inhibits melanogenesis in B16F10 mouse melanoma cells via glycogen synthase kinase $3 \beta$ phosphorylation. Int J Mol Med 36: 1353-1360, 2015.

22. Oh CT, Lee D, Koo K, Lee J, Yoon HS, Choi YM, Kwon TR and Kim BJ: Superoxide dismutase 1 inhibits alpha-melanocyte stimulating hormone and ultraviolet B-induced melanogenesis in murine skin. Ann Dermatol 26: 681-687, 2014.

23. Casanola-Martin GM, Le-Thi-Thu H, Marrero-Ponce Y, Castillo-Garit JA, Torrens F, Rescigno A, Abad C and Khan MT: Tyrosinase enzyme: 1 . An overview on a pharmacological target. Curr Top Med Chem 14: 1494-1501, 2014.

24. Smit N, Vicanova J and Pavel S: The hunt for natural skin whitening agents. Int J Mol Sci 10: 5326-5349, 2009.
25. Lee WJ, Bang S, Chung BY, Jung H, Oh ES and Chang SE: Inhibitory effects of N,N,N-trimethyl phytosphingosine-iodide on melanogenesis via ERK activation-mediated MITF degradation. Biosci Biotechnol Biochem 80: 121-127, 2015.

26. Ebanks JP, Wickett RR and Boissy RE: Mechanisms regulating skin pigmentation: The rise and fall of complexion coloration. Int J Mol Sci 10: 4066-4087, 2009.

27. Desmedt B, Rogiers V, Courselle P, De Beer JO, De Paepe K and Deconinck E: Development and validation of a fast chromatographic method for screening and quantification of legal and illegal skin whitening agents. J Pharm Biomed Anal 83: 82-88, 2013.

28. Kwak JY, Seok JK, Suh HJ, Choi YH, Hong SS, Kim DS and Boo YC: Antimelanogenic effects of luteolin 7-sulfate isolated from Phyllospadix iwatensis Makino. Br J Dermatol 175: 501-511, 2016.

29. Yoon WJ, Kim MJ, Koh HB, Lee WJ, Lee NH and Hyun CG: Effect of Korean Red Sea Cucumber (Stichopus japonicus) on Melanogenic Protein Expression in Murine B16 Melanoma. Int J Pharmacol 6: 37-42, 2010.

30. Slominski A, Moellmann G and Kuklinska E: L-tyrosine, L-dopa and tyrosinase as positive regulators of the subcellular apparatus of melanogenesis in Bomirski Ab amelanotic melanoma cells. Pigment Cell Res 2: 109-116, 1989.

31. Yanase $\mathrm{H}$, Ando $\mathrm{H}$, Horikawa $\mathrm{M}$, Watanabe $\mathrm{M}$, Mori $\mathrm{T}$ and Matsuda N: Possible involvement of ERK 1/2 in UVA-induced melanogenesis in cultured normal human epidermal melanocytes. Pigment Cell Res 14: 103-109, 2001.

32. Alam MB, Seo BJ, Zhao P and Lee SH: Anti-Melanogenic activities of heracleum moellendorffii via ERK1/2-Mediated MITF Downregulation. Int J Mol Sci 17: pii: e1844, 2016. 